\title{
Grau de consolidação de um intervalo sedimentar turbidítico na Bacia de Campos a partir de um modelo para velocidades sísmicas
}

\author{
Shayane Paes Gonzalez (COGE-ON/MCT) \& Jorge Leonardo Martins (COGE-ON/MCT)
}

Copyright 2016, SBGf - Sociedade Brasileira de Geofísica

Este texto foi preparado para a apresentação no VII Simpósio Brasileiro de Geofísica, Ouro Preto, 25 a 27 de outubro de 2016. Seu conteúdo foi revisado pelo Comitê Técnico do VII SimBGf, mas não necessariamente representa a opinião da SBGf ou de seus associados. É proibida a reprodução total ou parcial deste material para propósitos comerciais sem prévia autorização da SBGf.

\begin{abstract}
The prediction of $S$ wave velocity is present in different seismic applications. Therefore many models, both theoretical and empirical, have been developed for this purpose over the years. In this work an interactive method was used to estimate $S$ wave velocity, based on the BiotGassmann theory. The present study deals the application of the referred methodology to evaluate the relationship between seismic velocities and the degree of consolidation of the grains which compose a rock. The well log data arising from the well NA-01 located in Campos Basin were used in this work and the results obtained for the the turbiditic sedimentary interval were very robust confirming the stability of the method used.
\end{abstract}

\section{Introdução}

Os levantamentos sísmicos baseiam-se na propagação de ondas geradas artificialmente e, através destas, busca-se analisar a geologia presente em subsuperfície. Portanto, as velocidades de ondas sísmicas são largamente utilizadas para estudo de reservatórios de hidrocarbonetos. Todavia, muitas vezes, estes registros encontram-se ausentes, especialmente a velocidade da onda S, sendo a estimativa desta necessária em diversas aplicações, tais como: a modelagem sísmica, a substituição de fluidos, a análise AVO, entre outras (Castagna \& Backus, 1993).

Em vista da importância da temática exposta, Lee (2006) propôs um método interativo para a predição da velocidade da onda $S$ a partir da velocidade da onda $P$ medida, sob a consideração de que os efeitos de dispersão, de atenuação e a anisotropia podem ser desprezados. Tal técnica baseia-se na Teoria de BiotGassmann (Gassmann, 1951; Russel et al., 2001) e introduz equações empíricas para os módulos de compressibilidade $(\kappa)$ e cisalhamento $(\mu)$, sendo estes dependentes da porosidade dos sedimentos $(\phi)$ e do grau de consolidação entre os grãos $(\alpha)$.

Perfis geofísicos de poços foram utilizados para a realização da pesquisa e o modelo mencionado foi aplicado aos sedimentos turbidíticos do Reservatório Namorado situado no Campo de Namorado, Bacia de Campos, Rio de Janeiro, Brasil. Um estudo numérico foi realizado preliminarmente, através da análise da variação das velocidades sísmicas em função do parâmetro definido como grau de consolidação alfa, e, posteriormente, a velocidade da onda $S$ foi estimada. Os resultados obtidos foram bastante satisfatórios corroborando a estabilidade da metodologia proposta por Lee (2006).

\section{Metodologia}

As velocidades sísmicas para rochas saturadas com fluidos são expressas como (Russel et al., 2001; Oliveira \& Martins, 2011):

$$
\mathrm{V}_{\mathrm{P}, \mathrm{sat}}^{2}=\frac{\kappa_{\mathrm{sat}}+\frac{4}{3} \mu_{\mathrm{sat}}}{\rho_{\mathrm{b}}}
$$

e

$$
\mathrm{V}_{\mathrm{S}, \mathrm{sat}}^{2}=\frac{\mu_{\mathrm{sat}}}{\rho_{\mathrm{b}}},
$$

onde $\kappa_{\text {sat }}$ e $\mu_{\text {sat }}$ correspondem, respectivamente, ao módulo de compressibilidade e cisalhamento da rocha saturada e $\rho_{b}$ diz respeito à densidade efetiva. Lee (2006) baseou-se na teoria de Biot-Gassmann na qual $\kappa_{\text {sat }}$ é escrito da seguinte maneira:

$$
\kappa_{\mathrm{sat}}=\kappa_{\mathrm{d}}+\frac{\left[1-\kappa_{\mathrm{d}} / \kappa_{\mathrm{ma}}\right]^{2}}{\frac{\phi}{\kappa_{\mathrm{f}}}+\frac{(1-\phi)}{\kappa_{\mathrm{ma}}}-\frac{\kappa_{\mathrm{d}}}{\kappa_{\mathrm{ma}}^{2}}},
$$

sendo $\phi$ a porosidade total da rocha, $\kappa_{d}, \kappa_{f}$ e $\kappa_{\text {ma }}$ os módulos de cisalhamento da rocha seca, do fluido saturante e da matriz da rocha, respectivamente. De acordo com Pride et al. (2004) $\kappa_{\mathrm{d}}$ pode ser expresso como:

$$
\kappa_{\mathrm{d}}=\kappa_{\mathrm{ma}} \frac{1-\phi}{1+\alpha \phi}
$$

na qual $\alpha$ representa o grau de consolidação entre os grãos que compõem uma rocha. Por outro lado, para o módulo de cisalhamento, Lee (2006) sugere a relação subsequente:

$$
\mu_{\mathrm{sat}}=\mu_{\mathrm{ma}} \frac{(1-\phi)(1+\alpha)}{1+(1+\phi) \alpha+2 \phi \alpha^{2}} \text {. }
$$

Substituindo as equações (3), (4) e (5) nas relações (1) e (2) são obtidos modelos petrofísicos para as velocidades da onda P e S: 


$$
\begin{gathered}
\mathrm{V}_{\mathrm{P}, \mathrm{sat}}^{2}(\alpha)=\frac{1-\phi}{\rho_{\mathrm{b}}}\left\{\frac{1}{1+\alpha \phi}\right. \\
{\left[1+\frac{\phi(1+\alpha)^{2}}{(1-\phi)^{2} \alpha+(1-\phi)(1+\alpha \phi)^{\mathrm{K}_{\mathrm{ma}} / \mathrm{K}_{\mathrm{f}}}}\right] \kappa_{\mathrm{ma}}} \\
\left.\frac{4}{3} \mu_{\mathrm{ma}} \frac{(1+\alpha)}{\left[1+(1+\phi) \alpha+2 \phi \alpha^{2}\right]}\right\}
\end{gathered}
$$

e

$$
\mathrm{V}_{\mathrm{S}, \text { sat }}^{2}(\alpha)=\frac{1-\phi}{\rho_{\mathrm{b}}}\left[\frac{(1+\alpha)}{1+(1+\phi) \alpha+2 \phi \alpha^{2}}\right] \mu_{\mathrm{ma}} .
$$

Conforme a teoria acima exposta, Lee (2006) propôs uma metodologia para a estimativa da velocidade da onda $S$, através da seguinte minimização:

$$
\mathrm{V}_{\mathrm{p}}{ }^{*}(\alpha)-\mathrm{V}_{\mathrm{p}}{ }^{\mathrm{m}} \approx 0
$$

sendo $V_{p}{ }^{*}(\alpha)$ e $V_{p}{ }^{m}$ a velocidade da onda $P$ calculada pela Teoria de Biot-Gassmann e a velocidade da onda $P$ medida, nesta ordem. Substituindo a equação (6) na relação demonstrada acima obtém-se:

$$
\begin{gathered}
\mathrm{K}_{\mathrm{ma}}\left\{\frac{\phi(1+\alpha \phi)^{2}}{(1+\alpha \phi)\left[\frac{\mathrm{K}_{\mathrm{ma}}}{\mathrm{K}_{\mathrm{f}}}(1+\alpha \phi)+(1-\phi) \alpha\right]}\right\} \\
+\mathrm{K}_{\mathrm{ma}}\left(\frac{1-\phi}{1+\alpha \phi}\right)+\frac{4}{3} \mu_{\mathrm{ma}}\left[\frac{(1-\phi)(1+\alpha)}{1+(1+\phi) \alpha+2 \phi \alpha^{2}}\right] \\
-\rho_{\mathrm{b}}\left(\mathrm{V}_{\mathrm{p}}{ }^{\mathrm{m}}\right)^{2} \approx 0 .
\end{gathered}
$$

A seguir, o uso da metodologia proposta por Lee (2006) foi avaliada para estudar a variação do grau de consolidação nos sedimentos turbidíticos do Reservatório Namorado, Bacia de Campos.

\section{Resultados}

A metodologia descrita foi empregada aos dados provenientes do Poço NA-01 no Campo de Namorado e o intervalo de 3000 a $3150 \mathrm{~m}$ foi selecionado para a pesquisa.

A equação (9) requer que os valores da velocidade da onda $\mathrm{P}\left(\mathrm{V}_{\mathrm{p}}{ }^{\mathrm{m}}\right)$, da densidade efetiva $\left(\rho_{b}\right)$, dos módulos de compressibilidade e cisalhamento $\left(\kappa_{\mathrm{ma}}, \kappa_{\mathrm{f}}\right.$ e $\left.\mu_{\mathrm{ma}}\right)$ e da porosidade total $(\phi)$ sejam estabelecidos. Os dois primeiros são obtidos através de perfis geofísicos de poços, mais precisamente o perfil de vagarosidades sônicas da onda $\mathrm{P}$ e o perfil de densidades.

Já os módulos de compressibilidade e de cisalhamento foram considerados constantes e, neste trabalho, foram adotados: $\kappa_{m a}=36 \mathrm{GPa}, \mu_{m a}=45 \mathrm{GPa}$ e $\kappa_{f}=2,29 \mathrm{GPa}$.
Estes valores dizem respeito ao módulo de compressibilidade e cisalhamento de uma matriz composta por quartzo e ao módulo de compressibilidade do fluido de perfuração, nesta ordem.

Por outro lado, o cálculo da porosidade total $(\phi)$ foi realizado com o auxílio do perfil de densidades e da equação expressa a seguir:

$$
\phi=\frac{\rho_{\mathrm{ma}}-\rho_{\mathrm{b}}}{\rho_{\mathrm{ma}}-\rho_{\mathrm{f}}}
$$

onde $\rho_{\mathrm{ma}}$ e $\rho_{\mathrm{f}}$ são iguais a $2,65 \mathrm{~g} / \mathrm{cm}^{3}$ e $1,10 \mathrm{~g} / \mathrm{cm}^{3}$ e, representam, as densidades da matriz composta por quartzo e do fluido saturante, respectivamente.

Após inserir os valores de todas as constantes $\left(\kappa_{\mathrm{ma}}, \kappa_{\mathrm{f}} \mathrm{e}\right.$ $\left.\mu_{\mathrm{ma}}\right)$, da porosidade total calculada $(\phi)$, da densidade efetiva $\left(\rho_{\mathrm{b}}\right)$ e da velocidade da onda $\mathrm{P}$ medida $\left(\mathrm{V}_{\mathrm{p}}{ }^{\mathrm{m}}\right)$ na equação (9), nota-se a relação de dependência existente entre o grau de consolidação $(\alpha)$ e 0 modelo para as velocidades sísmicas proposto por Lee (2006).

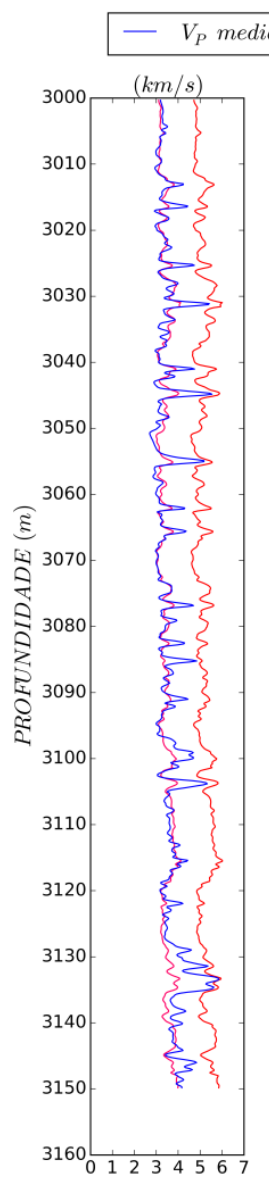

(a)

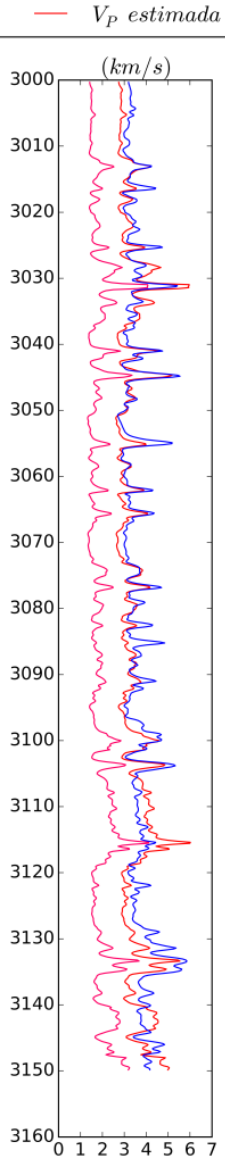

(b)

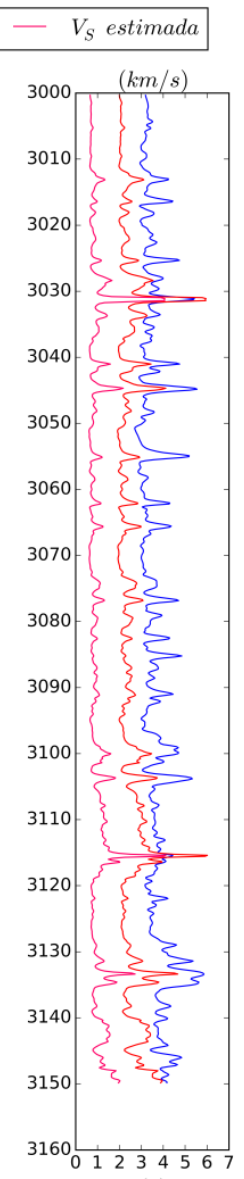

(c)
Figura 1: Testes para os valores do parâmetro alfa para os sedimentos turbidíticos do Reservatório Namorado. Em azul encontra-se a velocidade de onda $P$ medida. Já em vermelho e rosa as velocidades da onda $P$ e da onda $S$, respectivamente, calculadas com o parâmetro alfa igual a: (a) 1, (b) 10 e (c) 50. 
Por conseguinte, objetiva-se buscar as raízes da equação expressa em (9), ou seja, os valores de $\alpha$ que minimizem a relação. Neste trabalho o método interativo de Newton-Raphson foi empregado, visto que este apresenta grande rapidez e simplicidade para estimar as raízes de uma função.

Inicialmente foi realizado um breve estudo a respeito do comportamento de $V_{P}$ e $V_{S}$ com relação à variação do parâmetro $\alpha$ utilizando as equações (6) e (7). Desta forma, buscou-se compreender o melhor intervalo para alfa, isto é, os valores máximo e mínimo, de modo que as velocidades encontradas possuíssem significado físico correto.

A figura (1) exemplifica os testes realizados a priori. Em (a), o valor avaliado para $\alpha$ foi igual a 1 , sendo possível observar que a velocidades sísmicas assim calculadas foram superestimadas. Já em (b), para $\alpha$ igual a 10, os resultados obtidos para estimativa foram mais razoáveis, podendo-se verificar que o valor estimado para a onda $P$ se aproximou do valor medido. Por último, em (c), o valor de 50 foi atribuído a $\alpha$, resultando em subestimativas para a velocidade da onda $P$.

Isto posto, um valor inicial para $\alpha$ igual a 10 foi considerado para a construção do algoritmo do método de Newton-Raphson, assim como o intervalo para este parâmetro foi restringido de 0 a 50 . Uma vez finalizada a regressão linear, a verificação dos valores estimados para o parâmetro alfa foi realizada. Para tal, foram comparadas as velocidades preditas da onda P- ou seja, obtidas através dos valores de $\alpha$ e da equação (6)- e as velocidades da onda $\mathrm{P}$ verdadeiras.

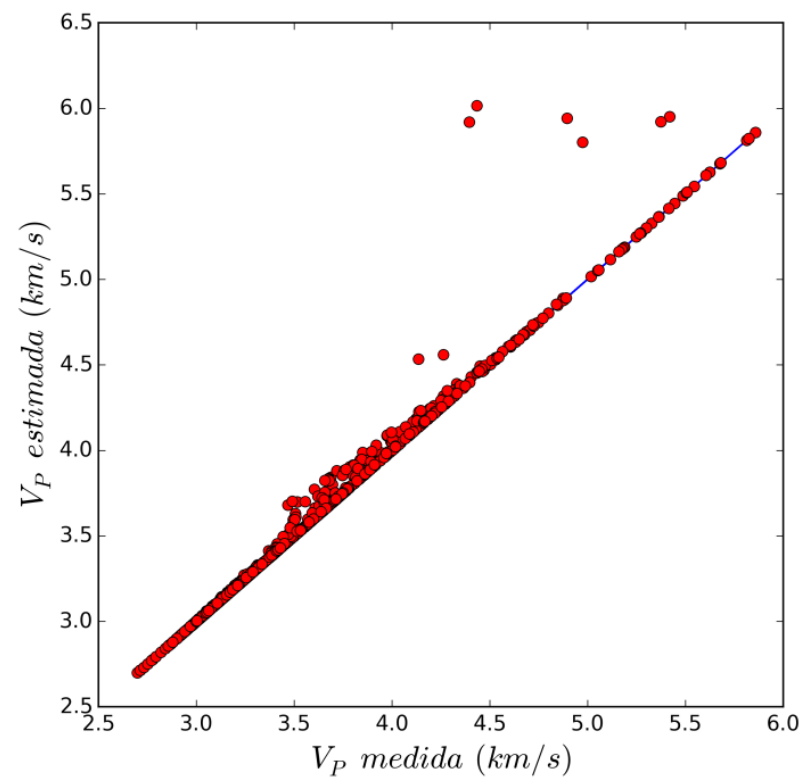

Figura 2: Comparação realizada entre a velocidade da onda $P$ medida e a velocidade da onda $P$ predita com a metodologia de Lee (2006) para os sedimentos turbidíticos do Reservatório Namorado.

A figura (2) mostra uma relação quase linear entre os dados preditos e os medidos, excetuando-se apenas alguns pontos. Visto que os valores para o parâmetro $\alpha$ estimado ajustaram-se de forma muito satisfatória, estes foram aplicados na equação (7) para que finalmente a estimativa da velocidade da onda $S$ fosse realizada.

A figura (3) mostra os resultados finais obtidos com os modelos descritos para as velocidades da onda $P$ e $S$ no intervalo de 150 m estudado. É possível observar em (a) que os resíduos absolutos (azul claro) gerados pela estimativa são pequenos, sendo a média, a variância $\left(\sigma^{2}\right)$ e o desvio padrão $(\sigma)$ apresentados na tabela (1).

Tabela 1: Dados estatísticos dos resíduos absolutos gerados.

\begin{tabular}{|c|c|c|}
\hline Média & Variância $\left(\boldsymbol{\sigma}^{2}\right)$ & $\begin{array}{c}\text { Desvio Padrão } \\
(\boldsymbol{\sigma})\end{array}$ \\
\hline 0,019 & 0,017 & 0,132 \\
\hline
\end{tabular}

Nas profundidades de aproximadamente 3030 e 3115 m, os erros destacam-se pelo aumento. Isto ocorre porque nestes locais a porosidade total calculada apresentou valores muito baixos, sendo esta a causa para as superestimativas de $V_{P}$ e $V_{S}$.
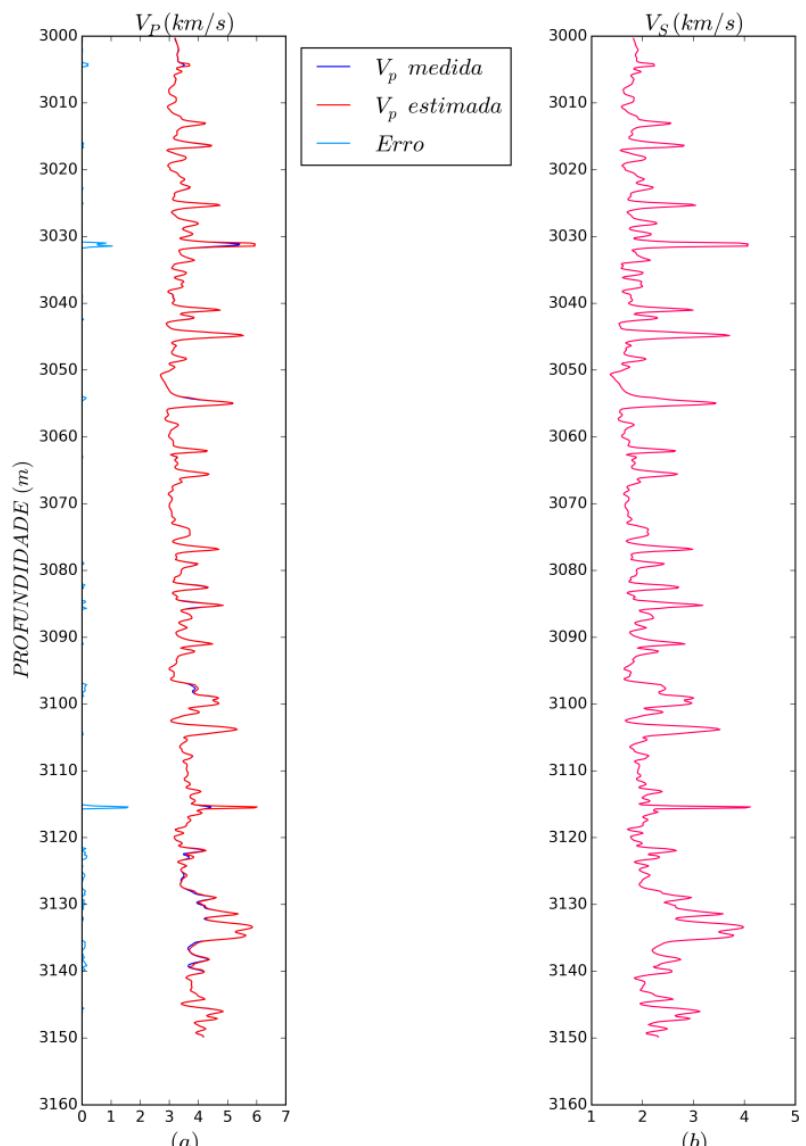

Figura 3: Velocidades sísmicas para o poço NA01. (a) Em azul encontra-se a velocidade da onda $P$ medida, em vermelho a velocidade da onda P predita com a metodologia de Lee (2006) e em azul claro os resíduos absolutos. (b) Velocidade da onda estimada com a metodologia de Lee (2006). 
A figura (4) mostra a relação existente entre as propriedades $\phi$ e $\alpha$, sendo notório que o aumento da primeira acarreta a diminuição da segunda. $O$ resultado obtido é esperado, visto que quanto maior o grau de consolidação entre os grãos que compõem uma rocha, menor será a porosidade total desta. Portanto, torna-se evidente a grande estabilidade existente na metodologia utilizada.

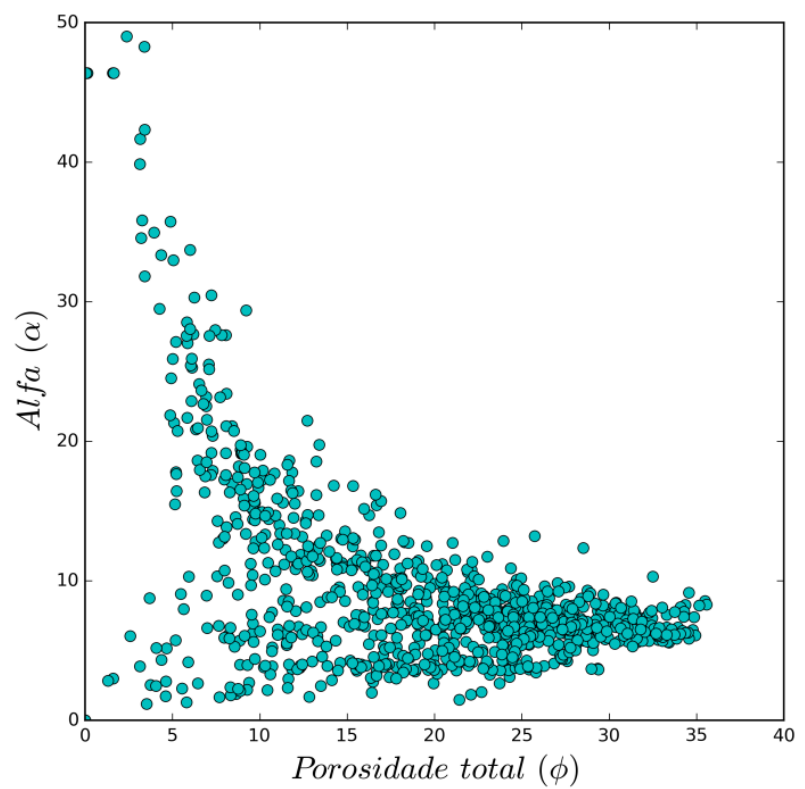

Figura 4: Valores estimados para o grau de consolidação entre os grãos e porosidade total para os sedimentos turbidíticos do Reservatório Namorado.

\section{Discussão e Conclusões}

A metodologia proposta por Lee (2006) ao ser aplicada aos dados provenientes do poço NA-01, no Campo de Namorado, demonstrou grande robustez. Os modelos utilizados para a estimativa das velocidades sísmicas apresentaram significativa estabilidade, visto a grande semelhança observada na comparação entre as velocidades da onda $P$ medidas e preditas. A relação existente entre os parâmetros de consolidação $\phi$ e $\alpha$ também corroborou para credibilidade do método, uma vez que o aumento da porosidade acompanhou a diminuição do grau de consolidação entre os grãos (valores de $\alpha$ estimados). Em suma, pelo aqui exposto, pode-se afirmar que é possível realizar a estimativa da velocidade da onda $S$ através do vínculo existente entre os módulos (de compressibilidade e cisalhamento) de uma rocha e a velocidade da onda $P$.

\section{Agradecimentos}

Jorge L. Martins recebe o apoio do CNPq, Cons. Nac. de Desenv. Científico \& Tecnológico, através de uma bolsa de produtividade em pesquisa $\mathrm{PQ}$ (proc. 302.480/-2011$0)$. O CNPq-MCTI apoia Shayane P. Gonzalez através de uma bolsa de pesquisa para desenvolvimento da dissertação no curso de PG em Geofísica do Observatório Nacional.

\section{Referências}

Castagna, J. \& Backus, M., 1993. Offset-Dependent Reflectivity: Theory and Practice of AVO Analysis, Society of Exploration Geophysicists.

Gassmann, F., 1951. Elastic waves through a packing of spheres, Geophysics, vol.16, p.673-685.

Lee, M. W., 2006. A simple method of predicting S-wave velocity. Geophysics, vol. 71, n. 6, p. F161-F164.

Oliveira, J. Sampaio, Martins, J. L., 2011. Spatial variation of angle-dependent impedance through a thin-layered oil reservoir. Revista Brasileira de Geofísica, vol. 29, n. 4.

Pride, S. R., Berryman, J.G. \& Harris, J.M., 2004. Seismic attenuation due to wave-induced flow. Journal of Geophysical Research: Solid Earth 109.B

Russell, B. H., Hedlin, K., Hilterman, F. J. \& Lines, L. R., 2001. Fluid-property discrimination with AVO: A BiotGassmann perspective, CREWES Research Report, vol. 13, p.403-419. 